\title{
Armchair nanoribbons of silicon and germanium honeycomb structures
}

\author{
S. Cahangirov, ${ }^{1}$ M. Topsakal, ${ }^{1}$ and S. Ciraci ${ }^{1,2, *}$ \\ ${ }^{1}$ UNAM-Institute of Materials Science and Nanotechnology, Bilkent University, Ankara 06800, Turkey \\ ${ }^{2}$ Department of Physics, Bilkent University, Ankara 06800, Turkey
}

(Received 25 January 2010; revised manuscript received 6 May 2010; published 25 May 2010)

\begin{abstract}
We present a first-principles study of bare and hydrogen passivated armchair nanoribbons of the puckered single layer honeycomb structures of silicon and germanium. Our study includes optimization of atomic structure, stability analysis based on the calculation of phonon dispersions, electronic structure, and the variation in band gap with the width of the ribbon. The band gaps of silicon and germanium nanoribbons exhibit family behavior similar to those of graphene nanoribbons. The edges of bare nanoribbons are sharply reconstructed, which can be eliminated by the hydrogen termination of dangling bonds at the edges. Periodic modulation of the nanoribbon width results in a superlattice structure which can act as a multiple quantum well. Specific electronic states are confined in these wells. Confinement trends are qualitatively explained by including the effects of the interface. In order to investigate wide and long superlattice structures we also performed empirical tight-binding calculations with parameters determined from $a b$ initio calculations.
\end{abstract}

DOI: 10.1103/PhysRevB.81.195120

PACS number(s): 73.22.-f, 63.22.- m, 61.48.De

\section{INTRODUCTION}

Graphene, a two-dimensional (2D) honeycomb structure of single layer of carbon atoms, has been synthesized and established as a material with wide range of unusual properties. ${ }^{1-3}$ It is a semimetal with a band profile having linear dispersion near the Fermi level, ${ }^{4}$ which attributes to its electrons a massless Dirac fermion behavior. Quasi-onedimensional (1D) derivatives of graphene, called graphene nanoribbons, were also produced recently. ${ }^{5,6}$ Graphene nanoribbons are semiconductors having interesting electronic properties depending on their geometry. These properties can be used to fabricate nanodevices as field effect transistors, spin valves, multiple quantum wells, etc. ${ }^{7-11}$

2D honeycomb structures and 1D nanotubes of $\mathrm{Si}$ and $\mathrm{Ge}$ were studied earlier. ${ }^{12,13}$ Stringent stability tests have recently shown that $2 \mathrm{D}$ honeycomb structures of $\mathrm{Si}$ and $\mathrm{Ge}$ can be found stable in a slightly buckled geometry. ${ }^{14}$ These structures have similar properties as graphene and thus carry the potential of being used in the similar applications. ${ }^{15} \mathrm{Com}-$ pared to graphene, the interatomic distance is larger in $\mathrm{Si}$ and $\mathrm{Ge}$, so the diminished $\pi-\pi$ overlaps cannot maintain the planar stability anymore. Eventually, the $s p^{2}$ hybrid orbitals are slightly dehybridized to form $s p^{3}$-like orbitals, which in turn results in a puckered structure. ${ }^{16}$ Freestanding graphene sheets and nanoribbons can be produced spontaneously, but it is not the case for $2 \mathrm{D}$ honeycomb structures of $\mathrm{Si}$ (silicene) and Ge. However, there are plenty experimental work on growth of $\mathrm{Si}$ nanoribbons especially on $\mathrm{Ag}$ surface. ${ }^{17,18}$ These highly metallic nanoribbons are formed by selforganization and have straight, atomically perfect, and massively parallel structures. ${ }^{18}$ The electronic structure of Si nanoribbons on $\mathrm{Ag}$ surface was also investigated theoretically. ${ }^{19}$

This paper reveals the atomic and electronic structure of armchair Si and Ge nanoribbons. Stability analysis, based on the calculation of the phonon dispersions via the force constant method, was performed. Energy band structure calculated by first-principles density-functional theory (DFT) was used to generate the parameters of the tight-binding model. It is found that armchair nanoribbons of $\mathrm{Si}$ and $\mathrm{Ge}$ are stable and their band gap vary with their width displaying a family behavior. ${ }^{20,21}$ Formation of multiple quantum well structure in superlattices consisting of periodically repeated junctions of nanoribbons having different widths was also investigated in detail. A 1D model was proposed to understand the effect of the interface in superlattice structures. It is found that specific electronic states are confined in these superlattice structures. The interface effects, which can explain unexpected confinements, are revealed.

\section{METHODS}

We have performed first-principles plane-wave calculations within local density approximation (LDA) (Ref. 22) using projector augmented wave (PAW) potentials. ${ }^{23}$ All structures are treated within supercell geometry using the periodic boundary conditions. A plane-wave basis set with kinetic energy cutoff of $300 \mathrm{eV}$ is used. In the self-consistent potential and total energy calculations, the Brillouin zone (BZ) is sampled by $(15 \times 1 \times 1)$ special $\mathbf{k}$ points. All atomic positions and lattice constants are optimized by minimization of the total energy and atomic forces. The vacuum separation between the nanoribbons in the adjacent unit cells is taken to be at least $10 \AA$. The convergence for energy is chosen as $10^{-5} \mathrm{eV}$ between two steps, and the maximum HellmannFeynman forces acting on each atom is less than $0.02 \mathrm{eV} / \AA$ upon ionic relaxation. Numerical plane-wave calculations have been performed by using VASP package. ${ }^{24,25}$ Phonon dispersions were obtained using the force constant method with forces calculated in a $(5 \times 1 \times 1)$ supercell. ${ }^{26,27} \mathrm{Cou}$ pling parameters of empirical tight-binding calculations are determined from the $a b$ initio results and are used to treat wide and long superlattice structures, comprising as many as $3600 \mathrm{Si}$ atoms.

\section{ATOMIC STRUCTURE AND STABILITY}

We first investigate the atomic structure of bare and hydrogen saturated armchair nanoribbons of $\mathrm{Si}$ and $\mathrm{Ge}$. The 
(a) Bare

(b) H passivated Bond Length $(\AA)$
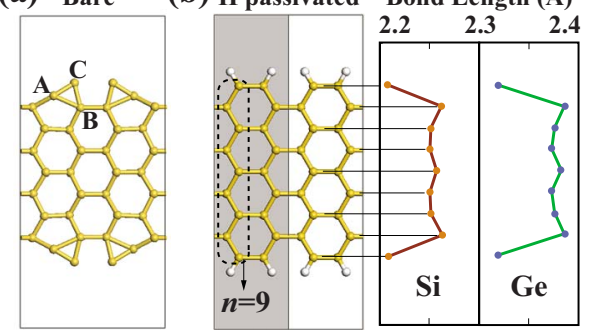

FIG. 1. (Color online) (a) Atomic structure of fully relaxed bare armchair Si nanoribbon of width $n=9$ (ASiNR-9). (b) Atomic structure and bond-length distribution of hydrogen saturated ASiNR-9. Similar pattern is observed in hydrogen saturated AGeNR-9. The primitive unit cell of ASiNR-9 is shaded. The zigzag chain of $\mathrm{Si}$ atoms perpendicular to the nanoribbon axis is delineated by the dashed lines.

ideal honeycomb structure is cut parallel to the nearestneighbor bonds to form an ideal bare nanoribbon with a certain width. Armchair nanoribbons are classified by counting the number, $n$, of $\mathrm{Si}$ (or $\mathrm{Ge}$ ) atoms forming a zigzag chain perpendicular to the cut direction. Accordingly, there are $2 n$ $\mathrm{Si}$ (or Ge) atoms in the primitive unit cell of an ideal bare armchair nanoribbon. This structure is treated by a supercell having periodic boundary condition in cut direction and a vacuum spacing in other directions. To lift the constraints imposed by $(1 \times 1)$ unit cell, we have used $(2 \times 1)$ supercell. Figure 1(a) presents the atomic structure of a sample bare armchair Si nanoribbon after structural relaxation. Here one can see a $(2 \times 1)$ reconstruction at the edges, which would be missed if $(1 \times 1)$ unit cell was used in the calculation. The edge reconstruction is reminiscent of the reconstruction of $\mathrm{Si}(100)-(2 \times 1)$ surface. In the latter case, two adjacent surface $\mathrm{Si}$ atoms each having two $s p^{3}$-dangling bonds come closer and form a new dimer bond using one $s p^{3}$-dangling bond from each atom. At the end, the number of $s p^{3}$ dangling bonds is halved and hence the energy is lowered through reconstruction. Similarly, in Fig. 1(a) A and B atoms come closer to form a bond. Since the nature of bonding is modified around B atom, the ABC triangle is bowed. At the end, the number of the $s p^{2}$ dangling bonds is halved. Note that this kind of reconstruction is not seen in bare armchair graphene nanoribbons. Moreover, in graphene and its nanoribbons all atoms lie in the same plane, while structures considered here are slightly buckled. The separation between adjacent atoms in the perpendicular direction to the plane is around 0.4 and $0.6 \AA$ for $\mathrm{Si}$ and $\mathrm{Ge}$ nanoribbons, respectively.

Figure 1(b) presents the atomic structure and bond-length distribution of a sample hydrogen saturated armchair silicon nanoribbons. In contrast to bare nanoribbons, saturation by hydrogen lifts the $(2 \times 1)$ reconstruction at the edges. In Fig. 1(b) there are $n=9 \mathrm{Si}$ atoms forming zigzag chain perpendicular to the nanoribbon axis and hence this armchair nanoribbon is classified as ASiNR-9. Accordingly the number of $\mathrm{Si}$ (or $\mathrm{Ge}$ ) atoms in the primitive unit cell is $2 n$. Note that the bond-length distribution is nearly uniform except a sudden decrease at the edges. This pattern was also observed in armchair graphene nanoribbons. ${ }^{20}$
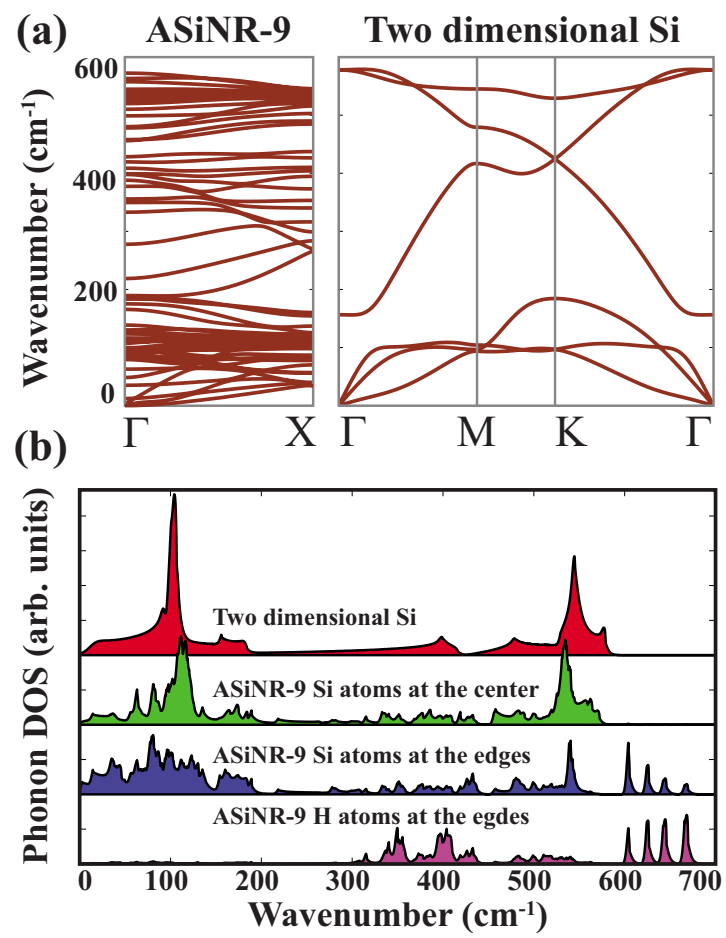

FIG. 2. (Color online) (a) Phonon dispersions calculated for hydrogen saturated ASiNR-9 and for 2D honeycomb structure of Si. States that appear above $600 \mathrm{~cm}^{-1}$ are related to $\mathrm{Si}-\mathrm{H}$ bonds of hydrogen saturated ASiNR-9 and were not shown. (b) Phonon DOSs of the hydrogen saturated ASiNR-9 projected to Si atoms at the center and at the edges and also to $\mathrm{H}$ atoms at the edges. DOS of $2 \mathrm{D}$ honeycomb structure of $\mathrm{Si}$ is also presented for comparison.

Figure 2(a) presents the phonon dispersion profile for hydrogen saturated ASiNR-9. Also phonon dispersion profile of 2D silicon, reproduced from Ref. 14, was shown for comparison. It is not possible to generate the dispersion profile of ASiNR by folding that of $2 \mathrm{D} \mathrm{Si}$, but the pattern is similar to that found in nanotube dispersion profiles obtained by zonefolding. ${ }^{28}$ In the phonon dispersion profile of hydrogen saturated ASiNR-9 all modes are real, except for some small imaginary frequencies calculated for the twisting acoustic mode, TW, near the $\Gamma$ point. This issue was faced earlier and was attributed to the limitations of the computational precision. $^{29}$ Thus, the structure is predicted to be stable. Computational cost of this calculation is very high, so we were not able to calculate the phonon dispersions for other ribbons. Nevertheless, all ASiNRs have very similar atomic configuration, and thus they are also expected to be stable. The phonon dispersion profile of $2 \mathrm{D} \mathrm{Ge}$ is similar to that of Si. ${ }^{16}$ But in Ge structure the acoustic and optic modes are well separated. Also due to softer bonds the wave numbers of Ge structure is halved compared to Si. Thus AGeNRs are also expected to be stable, while exhibiting the mentioned differences.

Phonon densities of states (DOSs) of hydrogen saturated ASiNR-9 projected to atoms at different locations in the nanoribbon are presented in Fig. 2(b). Shown is also DOS of the 2D Si honeycomb structure in the same figure. DOS projected on $\mathrm{Si}$ atoms at the center of the nanoribbon is very similar to that of the $2 \mathrm{D} \mathrm{Si}$. As the width of the nanoribbon 


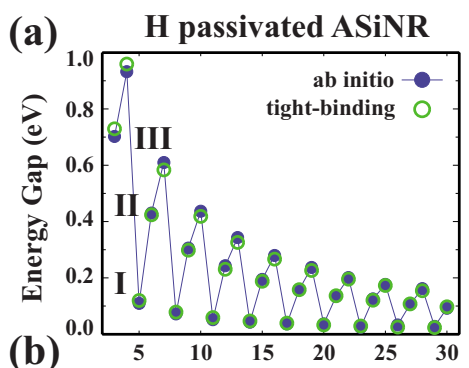

\section{H passivated AGeNR}
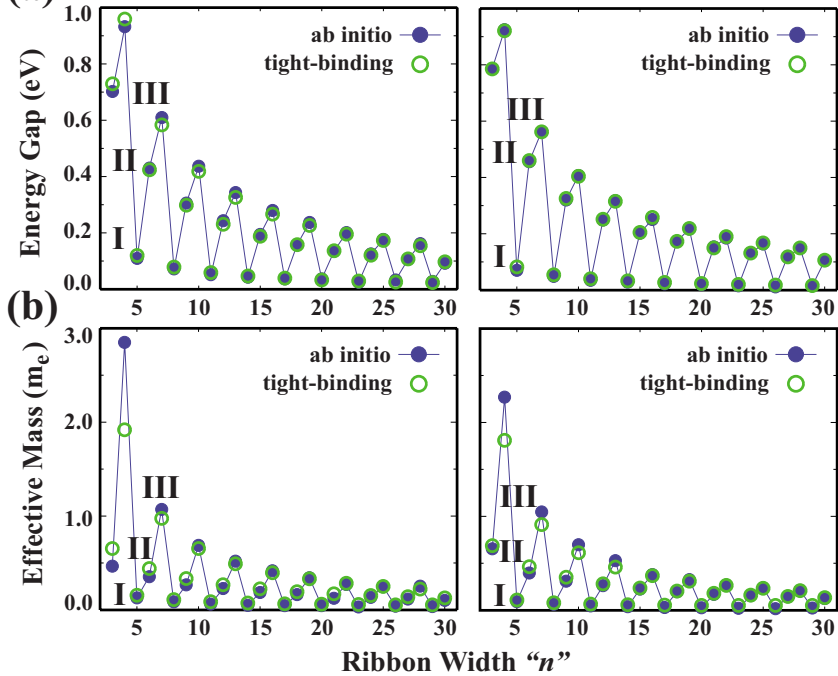

FIG. 3. (Color online) Calculated (a) energy gap and (b) effective mass versus ribbon width, $n$, for hydrogen saturated $\mathrm{Si}$ and $\mathrm{Ge}$ armchair nanoribbons. Filled circles indicate the $a b$ initio results while empty circles stand for the results of the tight-binding fitting. The fitting is performed using only the energy gap data. Parameters found from this fitting were used to generate the tight-binding effective mass data. In each panel three branches are observed and named in increasing order of band gaps and effective masses as families I, II, and III.

increases, this similarity is expected to be enhanced. However, DOS projected on Si atoms at the edges deviate from that corresponding to 2D Si. Especially, four optical peaks above $600 \mathrm{~cm}^{-1}$ are clearly originating from Si-H bonds at the edges. Also modes originating from short $\mathrm{Si}-\mathrm{Si}$ bonds at the edges cause changes in DOS below $600 \mathrm{~cm}^{-1}$.

\section{ELECTRONIC STRUCTURE}

In this section, we investigate the electronic structure of hydrogen saturated $\mathrm{Si}$ and $\mathrm{Ge}$ nanoribbons. Nanoribbons having widths from $n=3$ to 30 were investigated. Figure 3 presents the variation in energy gaps and effective masses with the ribbon width given in terms of $n$. Band gaps are direct and located at the $\Gamma$. One can see three branches with decaying profiles originating from the quantum size effect. Here in ascending order of the band gaps, we would like to name these branches with widths $n=3 k+2,3 k$, and $3 k+1$ as family I, II, and III, respectively, where $k$ is an integer. This "family behavior" was also observed in armchair graphene nanoribbons. ${ }^{20}$ This trend is explained by foldings of infinite graphene band profile, which is easily understood by using a tight-binding model. The simplest possible model is to assume that only the first nearest-neighbors interact and have equal hopping parameters with self-energies set to zero. But this model results in zero band gap for the members of the family I. Fortunately, this problem is fixed if a different parameter is used at the edges. ${ }^{20}$ As we mentioned in the previous section, the $\mathrm{Si}-\mathrm{Si}$ (and $\mathrm{Ge}-\mathrm{Ge}$ ) bond length is apparently smaller at the edges, which implies that edge bonds are stronger. That is why defining a different hopping parameter at the edges reflects the nature of the system better. Accordingly, we set the nearest-neighbor hoping integrals to be $t(1+\delta)$ at the edges and $t$ otherwise. In our model all self energies were set to zero. The tight-binding parameters were obtained by fitting to the first principles results. Results presented in Fig. 3(a) show that the model used here is successful in reproducing the DFT band-gap trends of both $\mathrm{Si}$ and Ge armchair nanoribbons. Since we did not make $G W$ correction to the band gaps, the nearest-neighbor hopping integrals, which are found to be $t=1.03 \mathrm{eV}$ for $\mathrm{Si}$ and $t$ $=1.05 \mathrm{eV}$ for $\mathrm{Ge}$, are expected to have larger values and should be taken as a qualitative result. However, the relative increase in the hopping integrals at the edges defined by $\delta$ can be taken as a quantitative result. We have found $\delta$ $=0.12$ and $\delta=0.08$ for $\mathrm{Si}$ and $\mathrm{Ge}$, respectively. Interestingly, the value found for $\mathrm{Si}$ is equal to that of reported value for the armchair graphene nanoribbons. To sum up, the tightbinding parameters determined for $\mathrm{Si}$ and $\mathrm{Ge}$ armchair nanoribbons are $t_{\mathrm{Si}}=1.03 \mathrm{eV}, t_{\mathrm{Ge}}=1.05 \mathrm{eV}, t_{\mathrm{Si}, \text { edge }}=1.15 \mathrm{eV}$, and $t_{\mathrm{Ge}, \text { edge }}=1.13 \mathrm{eV}$.

Figure 3(b) presents the effective masses of the first conduction band calculated by using the formula

$$
m^{*}=\hbar^{2}\left(\frac{\partial^{2} E(k)}{\partial k^{2}}\right)^{-1},
$$

where $E(k)$ is calculated by using both DFT and tightbinding model mentioned above. Note that parameters of the tight-binding model are generated by using only the bandgap information at the $\Gamma$ point, but it can reproduce the second-order momentum derivative and thus the effective mass, which is in agreement with that of ab initio calculation. The deviation from this agreement is seen in the ultimately thin nanoribbons. This is because, in these nanoribbons the edges affect the rest of the structure and the tightbinding model cannot be applied with the same success. Note that, the effective mass trends are similar to the bandgap trends and show the family behavior. Similar trends in the effective mass were also observed in the armchair graphene nanoribbons. ${ }^{30}$

\section{SUPERLATTICES}

In this section we investigate the electronic structure of superlattices formed by periodic junction of ASiNRs which have different widths. The variation in energy gaps with the ribbon width causes these structures act as a multiple quantum well and is expected to lead to interesting device applications. In the past, superlattices of 2D GaAs-AlAs or Si-Ge heterostructures have been extensively studied to realize new generation electronic devices. Recently, superlattices of graphene have been synthesized experimentally. ${ }^{31}$ The transport characteristics and electron confinement have been revealed..$^{32}$ Here superlattices are formed by the junction of two different nanoribbons. In this study the lengths of constituent nanoribbons are taken to be equal. The unequal cases were studied for armchair nanoribbons of graphene. ${ }^{11}$ We let $l$ unit cell of a ribbon with the width of $n_{1}$ to make a perfect junction with $l$ unit cell of a ribbon with the width of $n_{2}$; once joined these structures form a supercell having the 

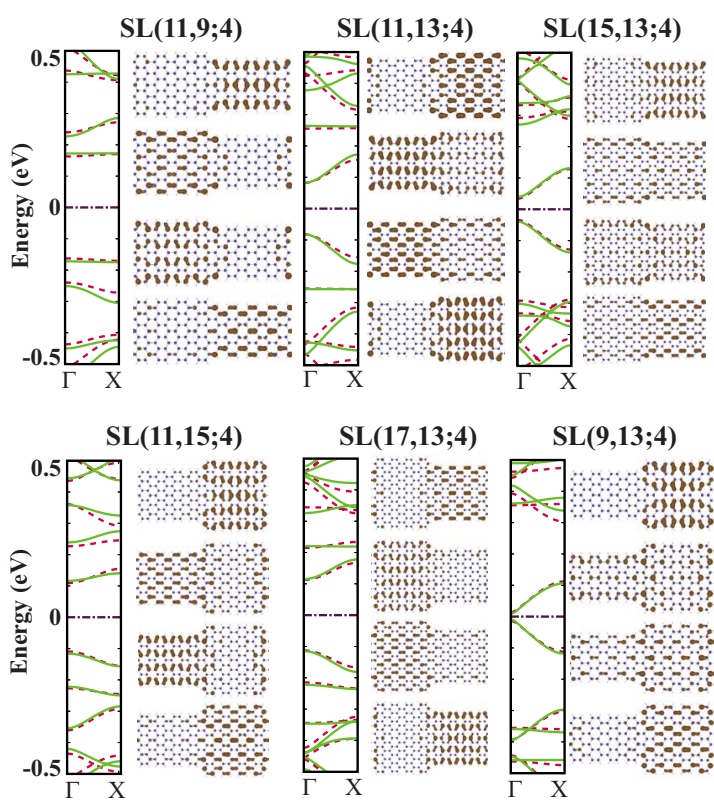

FIG. 4. (Color online) Band structure and charge density isosurface profiles for several superlattice structures. Solid (green) and dashed (red) lines stand for the $a b$ initio and tight-binding calculation results, respectively. Structures are labeled, as defined in the text, and are given on top of each structure. Top and bottom panels present the results for superlattice structures with $\Delta N=2$ and 4 , respectively. Constituent nanoribbons of superlattices that appear in the same column are members of the same family. Charge density isosurfaces calculated by ab initio technique around the $\Gamma$ point for two conduction and two valence band edge states are ordered in the similar manner as they appear on the energy band structure.

length of $2 l$. Here $n_{1}$ and $n_{2}$ are chosen to be odd so that the ribbons are joined symmetrically. Then the resulting structure is relaxed and the lattice constant of the whole structure is determined. This superlattice structure is labeled by its dimensions as $\operatorname{SL}\left(n_{1}, n_{2} ; l\right)$. The indices 1 and 2 are arranged in a way that a ribbon with a width $n_{1}$ has smaller band gap than a ribbon with a width $n_{2}$. The width difference $\Delta N$ $=n_{1}-n_{2}$ is defined for classification purpose.

Figure 4 presents the band structure and projected charge density isosurface plots for sample superlattices having $\Delta N$ $=2$ and 4 calculated by DFT. The band profile is nearly symmetric around the Fermi level and one can easily track the bonding and antibonding states from the charge density profile. As seen in the charge density plots, the band edge states of $\operatorname{SL}(11,9 ; 4)$ and $\operatorname{SL}(11,13 ; 4)$ superlattices are confined in the wide and narrow part, respectively. This can be explained by taking into account the band gaps of constituent ribbons of superlattice structures. Due to the symmetry between valence and conduction band edges of the constituent ribbons, the superlattice band lineup is always normal. As a result, the part having lower gap acts as a quantum well for electrons and holes. That is why, the electrons of $\operatorname{SL}(11,9 ; 4)$ and SL $(11,13 ; 4)$ structures are confined in the $n=11$ part, which for both superlattices is the part having the smaller energy gap.

The situation is also similar for the structures having $\Delta N=4$. Electrons of $\operatorname{SL}(17,13 ; 4)$ and $\operatorname{SL}(11,15 ; 4)$ are con- fined, respectively, in the wide and narrow part, which has the lower energy gap. Moreover, the superlattices constructed by the nanoribbons, which are members of the same family, have similar electronic structure. In Fig. 4 the superlattices having such common property are shown in the same column. Here one can see the similarity in the energy band profile of these structures. Another way to construct such structures is to increase the width of both constituent parts by $n=6 k$. To verify this, we have calculated the electronic structure of SL(17,15;4) and SL(17,19;4), which yielded in the similar results as that of $\operatorname{SL}(11,9 ; 4)$ and $\operatorname{SL}(11,13 ; 4)$, except that in former structures the confinements are less pronounced because the band-gap difference is lower.

We have to mention that, even though the structures $\operatorname{SL}(9,13 ; 4)$ and $\operatorname{SL}(15,13 ; 4)$ have a considerable band-gap difference between constituent parts, they do not have confinement neither in the wide nor in the narrow part. This is related to the interface effects, which will be discussed in a frame of another simple model.

Calculations for larger structures with $a b$ initio techniques are computationally too expensive. So we have used the tight-binding model mentioned before. In Fig. 4 one can see that the tight-binding model can reproduce the band edge profiles over the whole Brillouin zone. Moreover, the magnitudes of the eigenstates calculated by tight-binding model (not presented here mimics the projected charge density profile of a given state. To get a qualitative picture of how the large structures behave, the tight-binding model was used.

Figure 5 presents the energy gap and the band edge confinement strength trends generated by the tight-binding model for the superlattices having $n=9-31, \Delta N=2-6$, and $l=1-64$. The magnitude squares, $|\Psi|^{2}$, of conduction and valence band edge eigenfunctions generated by the tightbinding model are equal. The confinement percentage in the narrow (wide) part is defined as the sum of the magnitude squares of band edge eigenfunctions in the narrow (wide) part multiplied by $100 \%$, noting that the overall sum of the magnitude squares is normalized to 1 . In Fig. 5 we see that, in agreement with the $a b$ initio results presented in Fig. 4, the band edge states are confined in the narrow part for $\operatorname{SL}(11,15 ; 1)$ and $\operatorname{SL}(11,13 ; 1)$ structures and in the wide part for $\operatorname{SL}(11,9 ; 1)$ and $\operatorname{SL}(17,13 ; 1)$ structures. These confinements are enhanced as the lengths $l$ of the segments increase because the strength of the well and the barrier increase. The trends shown in Fig. 5 also confirm the statement that the superlattices composed of the nanoribbons from the same family behave similarly. For comparison, in each panel of the Fig. 5 structures having such similarity are shown in the same style. Also the corresponding family names are presented under the figure labels. The similarity can be seen in both confinement and the energy gap trends. For structures having $\Delta N=6$, the band edge states are always confined at the wide part. This is because the band gap for structures having $\Delta N=6$ is lower in the wide part, so the wide part acts as a quantum well.

In Fig. 5, one can see that there is no strong confinement in the wide or narrow part of $\operatorname{SL}(9,13 ; 1)$ and $\operatorname{SL}(15,13 ; 1)$ structures. This is also seen in the $a b$ initio results presented in Fig. 4. Moreover, the case is also true for the superlattices composed of the nanoribbons from the same family. Never- 

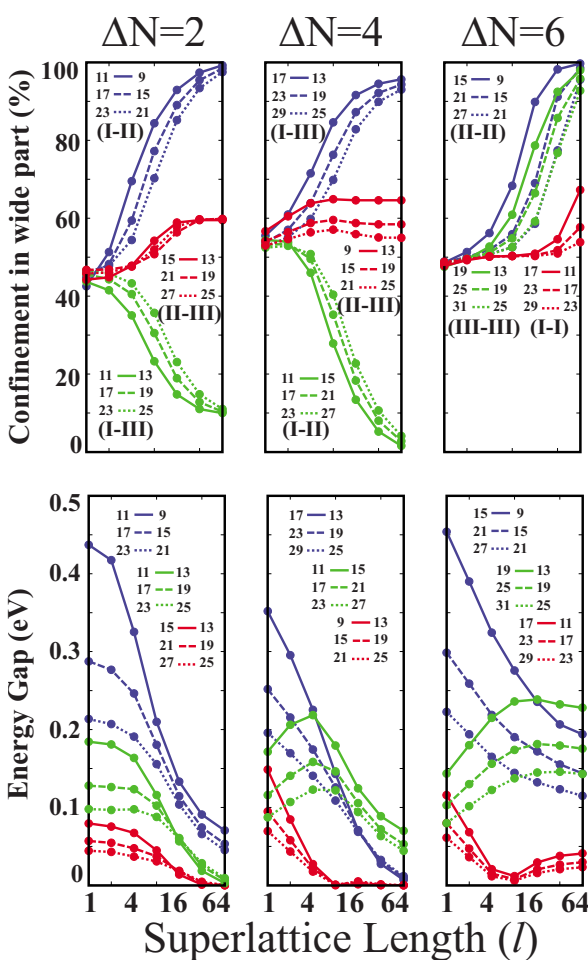

FIG. 5. (Color online) Variation in confinements and energy gaps of superlattices with the lengths of constituent parts. Results are derived by the tight-binding model described in the text. Numbers labeling the curves correspond to $n_{1}$ and $n_{2}$. The lengths, $l$, of the constituent parts are equal and shown in the logarithmic scale.

theless, this cannot be explained by the band-gap difference of the constituent nanoribbons because there are superlattices composed of the nanoribbons having similar band-gap difference but show strong confinement patterns. Actually, plotting the linear charge density of former structures along the periodic direction results in decaying profiles in both narrow and wide parts. Thus for these structures, the band edge states are localized at the interfaces. This means that the interface acts as a quantum well in these structures.

To consolidate the effects of the interface, we have to make an estimation about its effective energy gap and mass. For this purpose, we have chosen the energy gap and the effective mass of $\operatorname{SL}\left(n_{1}, n_{2} ; 1\right)$ structure to represent the effect of the interface between the nanoribbons with widths $n_{1}$ and $n_{2}$. This information was used in construction of a very simple 1D model, which qualitatively explains the confinement trends mentioned so far. Here the band edge state of a superlattice structure $\operatorname{SL}\left(n_{1}, n_{2} ; l\right)$ is modeled as a single electronic state which is under the influence of a periodic potential. The height of this potential is taken to be equal to the difference between the conduction band minimum and Fermi level of each region. The resulting profiles are shown for three different superlattice structures in Fig. 6(a). Here the interface effects are not included and the length parameter of each part is set to 64 times the length of a unit cell. Also the mass of electrons in each segment is modeled by the effective mass of a nanoribbon with a width $n$ of that segment. The magnitude square of the numerical solution of this system is presented with a light (green) line in the Fig. 6(b).

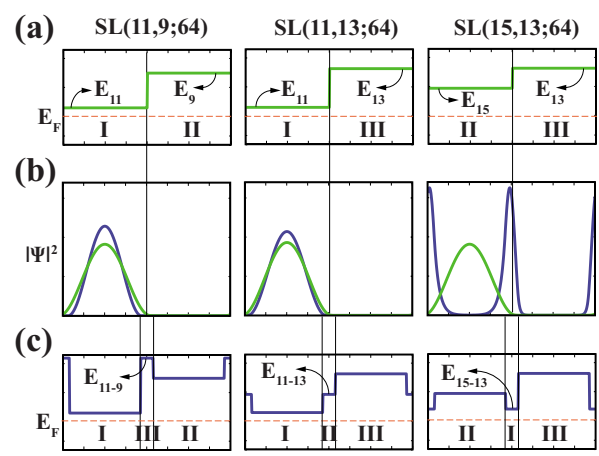

FIG. 6. (Color online) Model representation of three different superlattice structures. (a) Conduction band edge profile when interface effects are not included. Here the height of the potential in each side is determined by $E_{n}$, which is the difference between the conduction band edge minimum and Fermi level of a nanoribbon with a width $n$. Fermi levels of each structure are shown by the dashed lines. The family name corresponding to each side is written under these lines. (b) Magnitude squares $\left(|\Psi|^{2}\right)$ of solutions. Dark (blue) and light (green) lines represent solutions for the cases where interface effects are included and are not included, respectively. The thin line joining the panels (a) and (b) corresponds to the geometric interface of the superlattices. (c) Conduction band edge profile when interface effects are included. Here the height of the potential at the interface is determined by $E_{n_{1}-n_{2}}$, which is the difference between the conduction band edge minimum and Fermi level of $\operatorname{SL}\left(n_{1}, n_{2} ; 1\right)$ structure. The interfaces act as if they are composed of the families I, II, or III as written in that region.

To include the interface effects, the potential height of an arbitrary small region at the interface is changed to the difference between conduction band minimum and Fermi level of $\operatorname{SL}\left(n_{1}, n_{2} ; 1\right)$ structure. The dark (blue) lines in the Fig. 6(c) represent the new effective potential profiles. Solutions of these systems are given by dark (blue) lines in the Fig. 6(b).

Examining the changes induced by inclusion of the interface effects given in Fig. 6(b) one can deduce that in SL $(11,9 ; 64)$ and SL $(11,13 ; 64)$ structures the interface having large effective mass acts as a barrier which slightly enhances the confinement strength. In parallel to this, one can see in Fig. 4 that the charge densities are more localized in $\operatorname{SL}(11,9 ; 4)$ structure than in $\operatorname{SL}(11,13 ; 4)$ structure. Also the enhanced confinement of $\operatorname{SL}(11,9 ; 1)$ structure is seen in trends presented in Fig. 5. In SL $(15,13 ; 64)$ structure, however, the effect of the interface is dramatic. Since the effective energy gap of the interface region is smaller than that of the narrow and wide parts, it acts as a quantum well. As a result, we have a charge density confined in the interface region and decaying in the narrow and wide parts. The decay is sharper in the region where the effective potential is deeper and the mass is higher. This profile is similar to the one found by tight-binding model.

Trends found in this section can be summarized by very simple arguments; (i) if the superlattices are formed by two different families then the interface acts as if it is formed by a family different from both [see Fig. 6(c)]; (ii) in this case each part acts differently and electrons are confined in the part acting as the family I, which has the lowest band gap; 
(iii) if both sides are composed of the same family, then the electrons are confined at the wider part; and (iv) confinements are enhanced by increasing the lengths of the constituent parts.

\section{CONCLUSION}

We have investigated atomic structure, stability, and electronic properties of armchair silicon and germanium nanoribbons by performing first-principles calculations. The edges of bare armchair nanoribbons are reconstructed. The reconstruction is, however, removed by the hydrogen passivation of the edge atoms. It was shown that these nanoribbons exhibit the so-called family behavior which was explored also in graphene nanoribbons. A simple tight-binding model proposed for graphene nanoribbons was shown to work very well for silicon and germanium nanoribbons. Superlattices formed by periodic modulation of silicon nanoribbon widths were also investigated. Modulation of widths in the real space attributes these structures multiple quantum well properties. Specific electronic states are confined in these superlattice structures. Confinements increase with increasing the lengths of constituent parts of the superlattice. In general, the band edge states are confined in the part of superlattice, which have the smallest band gap by itself. This part can be narrow, wide, or the interface part of the superlattice. Superlattices composed of the nanoribbons which are members of the same family have similar electronic structure. Confinement patterns can be explained by inclusion of the interface effects in a frame of a simple 1D effective potential model. It is demonstrated that $\mathrm{Si}$ and Ge nanoribbons and superlattices constructed therefrom display features, which may be exploited in future nanodevices. *ciraci@fen.bilkent.edu.tr

${ }^{1}$ K. S. Novoselov, A. K. Geim, S. V. Morozov, D. Jiang, Y. Zhang, S. V. Dubonos, I. V. Grigorieva, and A. A. Firsov, Science 306, 666 (2004).

${ }^{2}$ Y. Zhang, Y.-W. Tan, H. L. Stormer, and P. Kim, Nature (London) 438, 201 (2005).

${ }^{3}$ C. Berger, Z. Song, X. Li, X. Wu, N. Brown, C. Naud, D. Mayou, T. Li, J. Hass, A. N. Marchenkov, E. H. Conrad, P. N. First, and W. A. de Heer, Science 312, 1191 (2006).

${ }^{4}$ K. S. Novoselov, A. K. Geim, S. V. Morozov, D. Jiang, M. I. Katsnelson, I. V. Grigorieva, S. V. Dubonos, and A. A. Firsov, Nature (London) 438, 197 (2005).

${ }^{5}$ M. Y. Han, B. Ozyilmaz, Y. Zhang, and P. Kim, Phys. Rev. Lett. 98, 206805 (2007).

${ }^{6}$ L. Jiao, L. Zhang, X. Wang, G. Diankov, and H. Dai, Nature (London) 458, 877 (2009).

${ }^{7}$ A. K. Geim and K. S. Novoselov, Nature Mater. 6, 183 (2007).

${ }^{8}$ X. Wang, Y. Ouyang, X. Li, H. Wang, J. Guo, and H. Dai, Phys. Rev. Lett. 100, 206803 (2008).

${ }^{9}$ Y. W. Son, M. L. Cohen, and S. G. Louie, Nature (London) 444, 347 (2006).

${ }^{10}$ M. Topsakal, H. Sevinçli, and S. Ciraci, Appl. Phys. Lett. 92, 173118 (2008).

${ }^{11}$ H. Sevinçli, M. Topsakal, and S. Ciraci, Phys. Rev. B 78, 245402 (2008).

${ }^{12}$ K. Takeda and K. Shiraishi, Phys. Rev. B 50, 14916 (1994).

${ }^{13}$ E. Durgun, S. Tongay, and S. Ciraci, Phys. Rev. B 72, 075420 (2005).

${ }^{14}$ S. Cahangirov, M. Topsakal, E. Aktürk, H. Şahin, and S. Ciraci, Phys. Rev. Lett. 102, 236804 (2009).

${ }^{15}$ Z. Y. Meng, T. C. Lang, S. Wessel, F. F. Assaad, and A. Muramatsu, Nature (London) 464, 847 (2010).

${ }^{16}$ H. Sahin, S. Cahangirov, M. Topsakal, E. Bekaroglu, E. Akturk, R. T. Senger, and S. Ciraci, Phys. Rev. B 80, 155453 (2009).

${ }^{17}$ H. Nakano, T. Mitsuoka, M. Harada, K. Horibuchi, H. Nozaki, N. Takahashi, T. Nonaka, Y. Seno, and H. Nakamura, Angew. Chem. 118, 6451 (2006).
${ }^{18}$ C. Léandri, G. Le Lay, B. Aufray, C. Girardeaux, J. Avila, M. E. Dàvila, M. C. Asensio, C. Ottaviani, and A. Cricenti, Surf. Sci. Lett. 574, L9 (2005); C. Léandri, H. Oughaddou, B. Aufray, J. M. Gay, G. Le Lay, A. Ranguis, and Y. Garreau, Surf. Sci. 601, 262 (2007); P. De Padova, C. Quaresima, P. Perfetti, B. Olivieri, C. Léandri, B. Aufray, S. Vizzini, and G. Le Lay, Nano Lett. 8, 271 (2008); P. De Padova, C. Léandri, S. Vizzini, C. Quaresima, P. Perfetti, B. Olivieri, H. Oughaddou, B. Aufray, and G. Le Lay, ibid. 8, 2299 (2008); A. Kara, C. Lèandri, M. E. Dàvila, P. De Padova, B. Ealet, H. Oughaddou, B. Aufray, and G. Le Lay, J. Supercond. Novel Magn. 22, 259 (2009); G. Le Lay, B. Aufray, C. Lèandri, H. Oughaddou, J. P. Biberian, P. De Padova, M. E. Dàvila, B. Ealet, and A. Kara, Appl. Surf. Sci. 256, 524 (2009).

${ }^{19}$ A. Kara, S. Vizzini, C. Lèandri, B. Ealet, H. Oughaddou, B. Aufray, and G. Le Lay, J. Phys.: Condens. Matter 22, 045004 (2010).

${ }^{20}$ Y. W. Son, M. L. Cohen, and S. G. Louie, Phys. Rev. Lett. 97, 216803 (2006)

${ }^{21}$ L. Yang, C.-H. Park, Y.-W. Son, M. L. Cohen, and S. G. Louie, Phys. Rev. Lett. 99, 186801 (2007).

${ }^{22}$ D. M. Ceperley and B. J. Alder, Phys. Rev. Lett. 45, 566 (1980).

${ }^{23}$ P. E. Blöchl, Phys. Rev. B 50, 17953 (1994).

${ }^{24}$ G. Kresse and J. Hafner, Phys. Rev. B 47, 558 (1993).

${ }^{25}$ G. Kresse and J. Furthmuller, Phys. Rev. B 54, 11169 (1996).

${ }^{26} \mathrm{G}$. Kresse, J. Furthmuller, and J. Hafner, Europhys. Lett. 32, 729 (1995).

${ }^{27}$ D. Alfè, Comput. Phys. Commun. 180, 2622 (2009).

${ }^{28}$ O. Dubay and G. Kresse, Phys. Rev. B 67, 035401 (2003).

${ }^{29}$ M. Topsakal, S. Cahangirov, E. Bekaroglu, and S. Ciraci, Phys. Rev. B 80, 235119 (2009).

${ }^{30}$ H. Raza and E. C. Kan, Phys. Rev. B 77, 245434 (2008).

${ }^{31}$ C. Stampfer, J. Güttinger, F. Molitor, D. Graf, T. Ihn, and K. Ensslin, Appl. Phys. Lett. 92, 012102 (2008).

${ }^{32}$ B. Özyilmaz, P. J. Herrero, D. Efetov, and P. Kim, Appl. Phys. Lett. 91, 192107 (2007). 